\title{
Time-Evolution Contrast of Target MRI Using High-Stability Antibody Functionalized Magnetic Nanoparticles: An Animal Model
}

\author{
K. W. Huang, ${ }^{1,2}$ S. Y. Yang, ${ }^{3}$ H. E. Horng, ${ }^{4}$ J. J. Chieh, ${ }^{4}$ H. H. Chen, ${ }^{4}$ \\ C. C. Wu, ${ }^{5,6}$ J. H. Chen, ${ }^{7}$ I.T. Lin, ${ }^{8,9}$ C. C. Yang, ${ }^{3}$ and H. C. Yang ${ }^{10}$ \\ ${ }^{1}$ Department of Surgery \& Hepatitis Research Center, National Taiwan University Hospital, Taipei 100, Taiwan \\ ${ }^{2}$ Graduate Institute of Clinical Medicine, College of Medicine, National Taiwan University, Taipei 100, Taiwan \\ ${ }^{3}$ MagQu Co., Ltd., New Taipei City 231, Taiwan \\ ${ }^{4}$ Institute of Electro-Optical Science and Technology, National Taiwan Normal University, Taipei 116, Taiwan \\ ${ }^{5}$ Department of Internal Medicine, National Taiwan University Hospital, College of Medicine, National Taiwan University, \\ Taipei 100, Taiwan \\ ${ }^{6}$ Department of Primary Care Medicine, National Taiwan University, Taipei 100, Taiwan \\ ${ }^{7}$ Department of Material Engineering, Kun Shan University, Tainan City 710, Taiwan \\ ${ }^{8}$ Center for Molecular Imaging and Translational Medicine, Xiamen University, Xiamen 361, China \\ ${ }^{9}$ Graduate Institute of Electronics Engineering, National Taiwan University, Taipei 106, Taiwan \\ ${ }^{10}$ Department of Electro-Optical Engineering, Kun Shan University, Tainan City 710, Taiwan
}

Correspondence should be addressed to S. Y. Yang; syyang@magqu.com

Received 30 May 2014; Accepted 23 July 2014; Published 27 August 2014

Academic Editor: Yali Cui

Copyright $\odot 2014$ K. W. Huang et al. This is an open access article distributed under the Creative Commons Attribution License, which permits unrestricted use, distribution, and reproduction in any medium, provided the original work is properly cited.

In this work, high-quality antibody functionalized $\mathrm{Fe}_{3} \mathrm{O}_{4}$ magnetic nanoparticles are synthesized. Such physical characterizations as particle morphology, particle size, stability, and relaxivity of magnetic particles are investigated. The immunoreactivity of biofunctionalized magnetic nanoparticles is examined by utilizing immunomagnetic reduction. The results show that the mean diameter of antibody functionalized magnetic nanoparticles is around $50 \mathrm{~nm}$, and the relaxivity of the magnetic particles is 145 $(\mathrm{mM} \cdot \mathrm{s})^{-1}$. In addition to characterizing the magnetic nanoparticles, the feasibility of using the antibody functionalized magnetic nanoparticles for the contrast medium of target magnetic resonance imaging is investigated. These antibody functionalized magnetic nanoparticles are injected into mice bearing with tumor. The tumor magnetic-resonance image becomes darker after the injection and then recovers 50 hours after the injection. The tumor magnetic-resonance image becomes the darkest at around 20 hours after the injection. Thus, the observing time window for the specific labeling of tumors with antibody functionalized magnetic nanoparticles was found to be 20 hours after injecting biofunctionalized magnetic nanoparticles into mice. The biopsy of tumor is stained after the injection to prove that the long-term darkness of tumor magnetic-resonance image is due to the specific anchoring of antibody functionalized magnetic nanoparticles at tumor.

\section{Introduction}

Due to its nontoxicity, nanosized, high-stability, iron oxide magnetic nanoparticles have been applied to in vitro and in vivo medical applications. For in vitro medical applications, several groups have published papers about the immunoaasay using biofunctionalized magnetic nanoparticles as labeling markers [1-6]. These papers reveal that the magnetically labeled immunoassay show high sensitivity, low interference, and versatility in clinics $[7,8]$. The in vivo applications using magnetic nanoparticles may include the magnetic labeling on cells $[9,10]$, drug delivery $[11,12]$, hyperthermia $[13,14]$, 
and so forth. Another important application of magnetic nanoparticles in in vivo medicine is the contrast medium for magnetic resonance (MR) imaging [15-18].

From the metabolism point of view, the injected magnetic nanoparticles injected into beings are eventually digested with liver. It has been further demonstrated that only normal liver cells can digest the injected magnetic nanoparticles, while liver tumor cells can not absorb the injected magnetic nanoparticles. With the magnetic nanoparticles, the relaxation time of MR like $\mathrm{T}_{2}$ for normal liver cells is reduced. Thus, for $\mathrm{T}_{2}$-weighted MR imaging, the brightness of normal cells is reduced. However, the brightness of $\mathrm{T}_{2}$-weighted $\mathrm{MR}$ image for liver tumor cells remains unchanged. Therefore, by injecting magnetic nanoparticles into beings, the bright spots on liver MR image are regarded as tumors [19]. Nowadays, iron oxide magnetic nanoparticles are used as contrast medium in clinics for imaging liver tumors.

For other kinds of tumors, target MR imaging using biofunctionalized $\mathrm{Fe}_{3} \mathrm{O}_{4}$ magnetic nanoparticles is utilized [2022 ]. For a given kind of tumor, specific antigens are expressed by the tumor. Magnetic nanoparticles immobilized with antibodies against the antigens are able to bind with the tumor and attribute to the reduction in the relaxation times of MR. Thus, the MR image of the tumor becomes darker when compared with that of surrounding normal cells. Practically, as the antibody functionalized magnetic nanoparticles are injected to beings and are circulated to the tumor, both the tumor and surrounding normal cells are rich in magnetic nanoparticles. Thus, MR images of the tumor and surrounding normal cells become dark. It is impossible to differentiate the tumor from the surrounding normal cells. After a period of time, the tumor keeps the antibody functionalized magnetic nanoparticles, while the magnetic nanoparticles around the normal cells are washed away. During this period of time, the MR image of the tumor is darker than that of the surrounding normal cells. It is easy to find the tumor according to the MR image. But, the binding of the antibody functionalized magnetic nanoparticles with the tumor is not permanent. The bound magnetic nanoparticles with the tumor eventually are digested or washed away after certain period of time. The above descriptions imply that there exists a suitable time window for imaging the tumor using MR. However, the nanoparticles of the imaging time window of MR for target tumors are very rare.

In this work, the time-evolution of MR images of mice bearing with colorectal tumors is recorded after the injection of antibody functionalized $\mathrm{Fe}_{3} \mathrm{O}_{4}$ magnetic nanoparticles. Besides, the synthesis of antibody functionalized $\mathrm{Fe}_{3} \mathrm{O}_{4}$ magnetic nanoparticles is introduced. Since colorectal tumors express carcinoembryonic antigen (CEA), antibody against CEA is conjugated onto $\mathrm{Fe}_{3} \mathrm{O}_{4}$ magnetic nanoparticles. The characterizations, such as particle size, MR relaxivity, stability and magnetization, of these magnetic nanoparticles are examined.

\section{Experimental Details}

The protocol of synthesizing magnetic $\mathrm{Fe}_{3} \mathrm{O}_{4}$ nanoparticles was proposed by MagQu Co., Ltd. [23]. The flow chart for

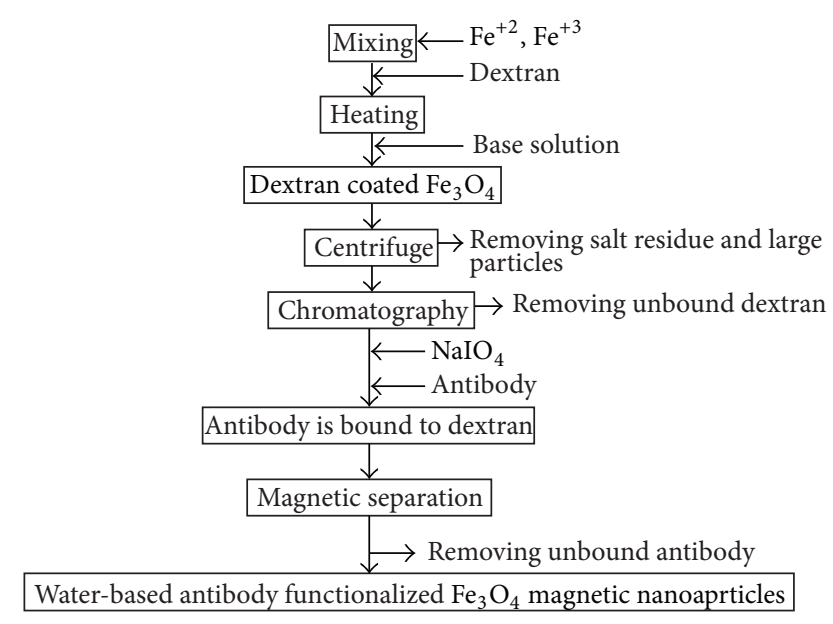

FIgURE 1: Flow chart of synthesizing antibody functionalized magnetic nanoparticles dispersed in PBS solution.

the synthesis of antibody functionalized magnetic nanoparticles is shown in Figure 1. A ferrite solution containing a stoichiometric ratio of $1: 2$ ferrous sulphate heptahydrate $\left(\mathrm{FeSO}_{4} \cdot 7 \mathrm{H}_{2} \mathrm{O}\right)$ and ferric chloride hexahydrate $\left(\mathrm{FeCl}_{3} \cdot 6 \mathrm{H}_{2} \mathrm{O}\right)$ was mixed with an equal volume of aqueous dextran, which acted as a surfactant for $\mathrm{Fe}_{3} \mathrm{O}_{4}$ particles dispersed in water. The mixture was heated to $70-90^{\circ} \mathrm{C}$ and titrated with strong base solution to form black $\mathrm{Fe}_{3} \mathrm{O}_{4}$ particles. Aggregates and excess unbound dextran were removed by centrifugation and gel filtration chromatography to obtain highly concentrated homogeneous magnetic fluid. The reagent (MF-DEX-0060, $\mathrm{MagQu}$ ) with desired magnetic concentration was obtained by diluting the highly concentrated magnetic fluid with pH-7.4 phosphate buffered saline (PBS) solution. To make antibodies against carcinoembryonic antigen (CEA) for colorectal cancer, that is, anti-CEA (10C-CR2014M5, Fitzgerald; AT-CEA, MagQu), bound to the dextran on the outmost shell of magnetic nanoparticles, $\mathrm{NaIO}_{4}$ solution was added into the magnetic solution to oxide dextran, which was then used to create aldehyde groups $(-\mathrm{CHO})$. Then, dextran can react with anti-CEA via the linking of $-\mathrm{CH}=\mathrm{N}-$. Thus, anti-CEA is bound covalently to dextran. Through magnetic separation, unbound anti-CEA was separated from the solution.

The size distribution of $\mathrm{Fe}_{3} \mathrm{O}_{4}$ magnetic nanoparticles biofunctionalized without/with anti-CEA was analyzed by using dynamic laser scattering (Nanotrac 150, Microtrac). The morphology of anti-CEA functionalized $\mathrm{Fe}_{3} \mathrm{O}_{4}$ magnetic nanoparticles is analyzed by using a scanning electronic microscope (JSM-6700F, Jeol). The relaxivity of MR for the reagent is measured by 3-T MR imaging instrument (Biospec System, Bruker).

To check the immunoreactivity of anti-CEA functionalized $\mathrm{Fe}_{3} \mathrm{O}_{4}$ magnetic nanoparticles, the technology so-called immunomagnetic reduction is used [24-26]. The working principle of immunomagnetic reduction (IMR) is illustrated in Figure 2. In IMR, antibody functionalized magnetic nanoparticles are under the actions of multiple ac magnetic fields. 


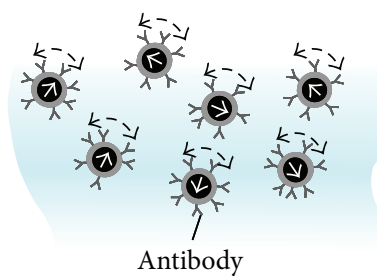

(a) Before association, $\chi_{\mathrm{ac}, \mathrm{o}}$

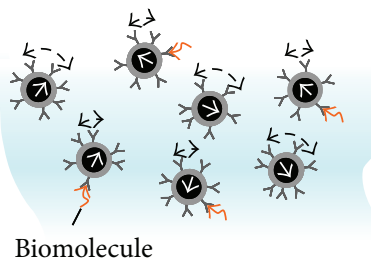

(b) After association, $\chi_{\mathrm{ac}, \phi}\left(<\chi_{\mathrm{ac}, \mathrm{o}}\right)$

FIGURE 2: Illustration for the mechanism of immunomagnetic reduction.

Thus, magnetic nanoparticles exhibit an ac magnetic susceptibility $\chi_{\mathrm{ac}}$. As the antibody functionalized magnetic nanoparticles are mixed with a solution with target biomolecules. Via the antibodies on magnetic nanoparticles, nanoparticles associate with target biomolecules. Before the association, magnetic nanoparticles exhibit an ac magnetic susceptibility, denoted by $\chi_{\mathrm{ac}, \mathrm{o}}$ in Figure 2(a). After the association, some magnetic nanoparticles become larger, which results in the reduction in the ac magnetic susceptibility. The ac magnetic susceptibility of magnetic nanoparticles after biomoleculeparticle association is denoted by $\chi_{\mathrm{ac}, \phi}$ in Figure 2(b). $\chi_{\mathrm{ac}, \phi}$ is smaller than $\chi_{\mathrm{ac}, \mathrm{o}}$. The reduction percentage in $\chi_{\mathrm{ac}}$ of magnetic nanoparticles due to the biomolecule-particle association is defined as IMR signal as follows:

$$
\operatorname{IMR}(\%)=\frac{\left(\chi_{\mathrm{ac}, \mathrm{o}}-\chi_{\mathrm{ac}, \phi}\right)}{\chi_{\mathrm{ac}, \mathrm{o}}} \times 100 \% .
$$

The ac magnetosusuceptometer (XacPro-E, MagQu) is used to monitor the time dependent $\chi_{\mathrm{ac}}$ of the anti-CEA functionalized $\mathrm{Fe}_{3} \mathrm{O}_{4}$ magnetic nanoparticles after mixing with CEA solution. A significant reduction in the ac magnetic susceptibility of magnetic nanoparticles can be found once CEA molecules bind with active anti-CEA on magnetic nanoparticles. Furthermore, more reduction in ac magnetic susceptibility is obtained for more CEA molecules. That is, the sample with higher CEA concentrations shows higher IMR signals. On the other hand, the activity of anti-CEA on magnetic nanoparticles fails; no significant IMR signal can be observed.

For implanting the colorectal tumors, the injections of the CT-26 cell line were processed through the skin on the backs of 8 -week-old mice. Three weeks later, $0.06 \mathrm{emu} / \mathrm{g}$ and $100 \mu \mathrm{L}$ of anti-CEA magnetic reagent were injected into the tail veins of five mice. Two groups of mice are used for examinations of MR imaging and Prussian blue $(\mathrm{PB})$ staining, respectively, as tabulated in Table 1 . Group 1 formed with two mice, numbered as mouse 1 and mouse 2, is examined using MR imaging instrument. The MR examination schedule was at the 0th, 18th, 30th, 48th, 72nd, and 96th hours for mouse 1 and at the 0th, 12th, 24th, and 46th hours for mouse 2. Here, 0th hour represents the time just before injection. Proving that the anti-CEA $\mathrm{Fe}_{3} \mathrm{O}_{4}$ magnetic nanoparticles were bound to the tumor tissue required determining the Fe using Prussian blue (PB) staining to examine the tumor tissue of Group 2, which is formed with mouse 3, mouse 4,

TABLE 1: Two groups of mice used for the examinations of MR imaging and PB staining.

\begin{tabular}{lcc}
\hline Group & Mouse number & Experiment \\
\hline 1 & 1 and 2 & Magnetic resonance (MR) imaging \\
2 & 3,4 , and 5 & Prussian blue (PB) staining \\
\hline
\end{tabular}

and mouse 5, which were euthanized at the 0th, 24th, and 98th hours, respectively. The tissue staining was processed (Laboratory Animal Center, National Taiwan University, Taipei, Taiwan), and the $\times 400$ magnification of the optical images was observed using a light microscope.

The 3-T MR imaging (Biospec System, Bruker) and a volume coil were used for $\mathrm{T}_{2}$-weighted images. In parallel with the arrangement of the anesthetized mouse, a long tube filled with deionized (DI) water was inserted as the intensity reference to dismiss the instrument drift at various times. Producing the coronal images of each entire mice body at $2 \mathrm{~mm}$ intervals required nearly 2 hours.

\section{Results and Discussion}

The distribution of particle diameter of dextran-coated $\mathrm{Fe}_{3} \mathrm{O}_{4}$ magnetic nanoparticles is analyzed using dynamic laser scattering. The results are shown in the upper part of Figure 3(a) with the schematically illustration of dextran-coated $\mathrm{Fe}_{3} \mathrm{O}_{4}$ magnetic nanoparticle. The mean diameter of dextran-coated $\mathrm{Fe}_{3} \mathrm{O}_{4}$ magnetic nanoparticles was found to be $42.80 \mathrm{~nm}$. After immobilizing anti-CEA on the dextran-coated $\mathrm{Fe}_{3} \mathrm{O}_{4}$ magnetic nanoparticles, the distribution of particle diameter is analyzed, as shown in the lower part of Figure 3(a). In the inset, the $\mathrm{Ys}$ on the dextran-coated $\mathrm{Fe}_{3} \mathrm{O}_{4}$ magnetic nanoparticle denote anti-CEA. The mean diameter of antiCEA functionalized $\mathrm{Fe}_{3} \mathrm{O}_{4}$ magnetic nanoparticles was found to be $51.3 \mathrm{~nm}$, which is larger than that of dextran-coated $\mathrm{Fe}_{3} \mathrm{O}_{4}$ magnetic nanoparticles by $8.5 \mathrm{~nm}$. Hence, the thickness of anti-CEA on the outmost layer of $\mathrm{Fe}_{3} \mathrm{O}_{4}$ magnetic nanoparticles is around $4.25 \mathrm{~nm}$. The shapes of the antibody functionalized magnetic nanoparticles are examined by using scanning electronic microscope and are shown in Figure 3(b). It is obvious that each particle is almost identical. Hence, the homogeneity of particle size is high.

To investigate the stability of the suspension of antibody functionalized $\mathrm{Fe}_{3} \mathrm{O}_{4}$ magnetic nanoparticles in PBS solution, the storing-period dependent mean diameter of nanoparticles is explored. In case of low-stability suspension, the magnetic nanoparticles initially suspended in individual in PBS solution agglomerate with each other as time goes by. An increase in the mean diameter of nanoparticles will be observed. On the other hand, if the mean diameter of nanoparticles was observed to be unchanged, the stability of suspension of magnetic nanoparticles in PBS solution was high. The experimental results are shown in Figure 4. The reagent of antibody functionalized $\mathrm{Fe}_{3} \mathrm{O}_{4}$ magnetic nanoparticles is stored at $2-8^{\circ} \mathrm{C}$. It is clear in Figure 4 that the mean diameter of antibody functionalized $\mathrm{Fe}_{3} \mathrm{O}_{4}$ magnetic nanoparticles in PBS solution remains around $50 \mathrm{~nm}$ for 7.7 -month storage. 

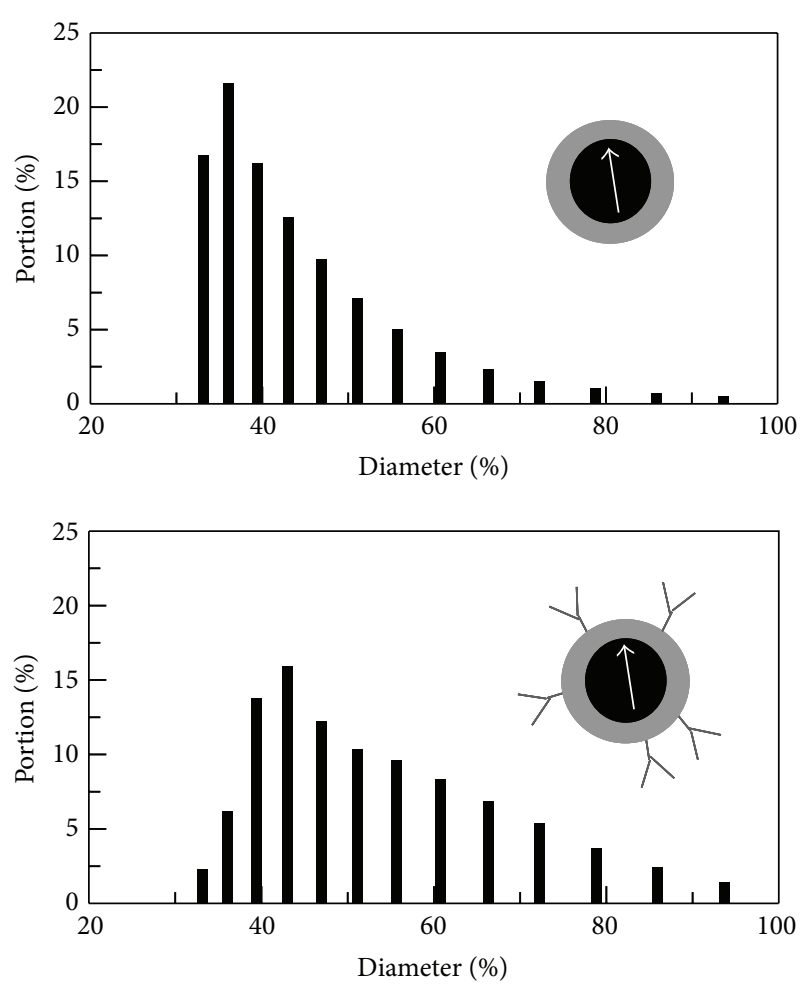

(a)

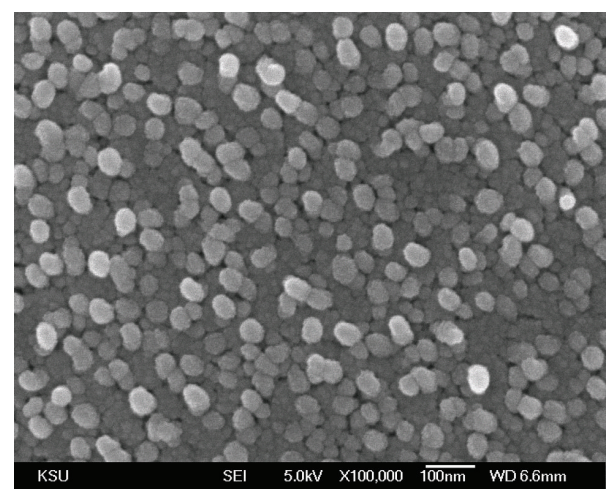

(b)

Figure 3: Analysis of particle diameter for $\mathrm{Fe}_{3} \mathrm{O}_{4}$ magnetic nanoparticles (a) without (upper) and with (lower) antibodies suspended in PBS solution using dynamic laser scattering and (b) with antibodies using scanning electronic microscope.

The results evidence the high-stability suspension of antibody functionalized $\mathrm{Fe}_{3} \mathrm{O}_{4}$ magnetic nanoparticles in PBS solution.

It is important to check the immunoreactivity of antiCEA as conjugated onto dextran-coated $\mathrm{Fe}_{3} \mathrm{O}_{4}$ functionalized magnetic nanoparticles. One of methods for this check is to observe the association between the anti-CEA functionalized $\mathrm{Fe}_{3} \mathrm{O}_{4}$ magnetic nanoparticles and CEA molecules in PBS solution. The method used in this experiment for observing the nanoparticle-CEA association is so-called immunomagnetic reduction [24-26]. The time dependent ac magnetic susceptibility $\chi_{\mathrm{ac}}$ of the reagent mixing with $5 \mathrm{ng} / \mathrm{mL}$ CEA

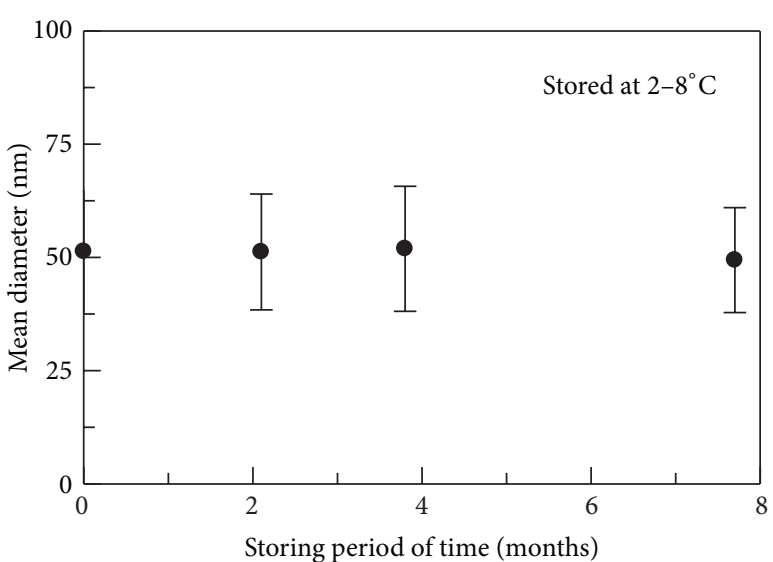

FIgURE 4: Storing-period dependent mean diameter of antibody functionalized $\mathrm{Fe}_{3} \mathrm{O}_{4}$ magnetic nanoparticles.

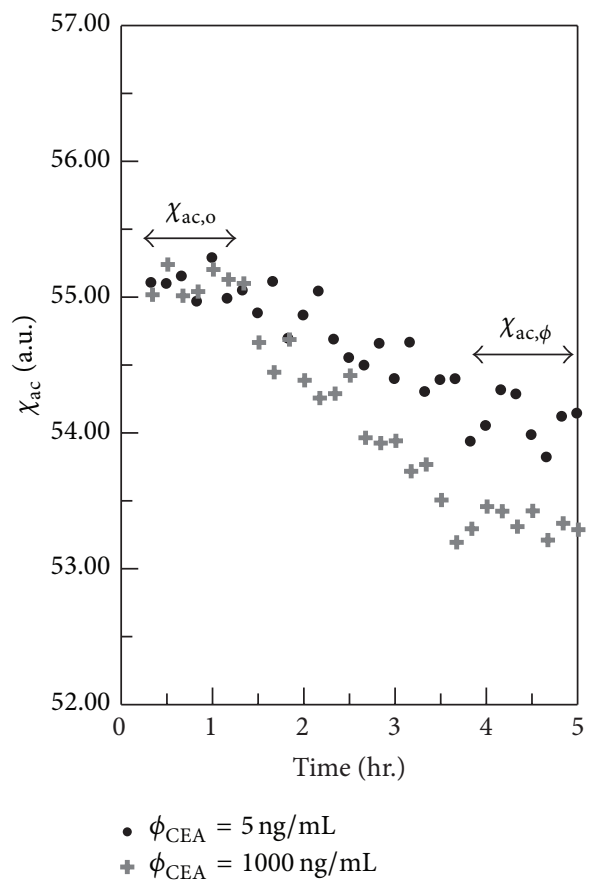

FIGURE 5: Time dependent ac magnetic susceptibility of reagent reacting with CEA in PBS solution of various CEA concentrations.

solution was recorded and shown with dots in Figure 5. It was found that $\chi_{\mathrm{ac}}$ of the reagent keeps unchanged just after mixing with the CEA solution, as labeled with $\chi_{\mathrm{ac}, \mathrm{o}}$ in Figure 5. After one hour, the $\chi_{\mathrm{ac}}$ of the reagent starts to reduce. The reduction in $\chi_{\mathrm{ac}}$ of the reagent is not terminated until 3.8 hours after the mix of the reagent and the CEA solution. The IMR signal via (1) was found to be $1.68 \%$. The reduction in $\chi_{\mathrm{ac}}$ of the reagent proves the association between the anti-CEA functionalized $\mathrm{Fe}_{3} \mathrm{O}_{4}$ magnetic nanoparticles and CEA molecules. As the CEA concentration is increased, say $1000 \mathrm{ng} / \mathrm{mL}$, the reduction in $\chi_{\mathrm{ac}}$ of the reagent is enhanced, as presented with cross symbols in Figure 5. The IMR signal for $1000 \mathrm{ng} / \mathrm{mL}$ CEA 


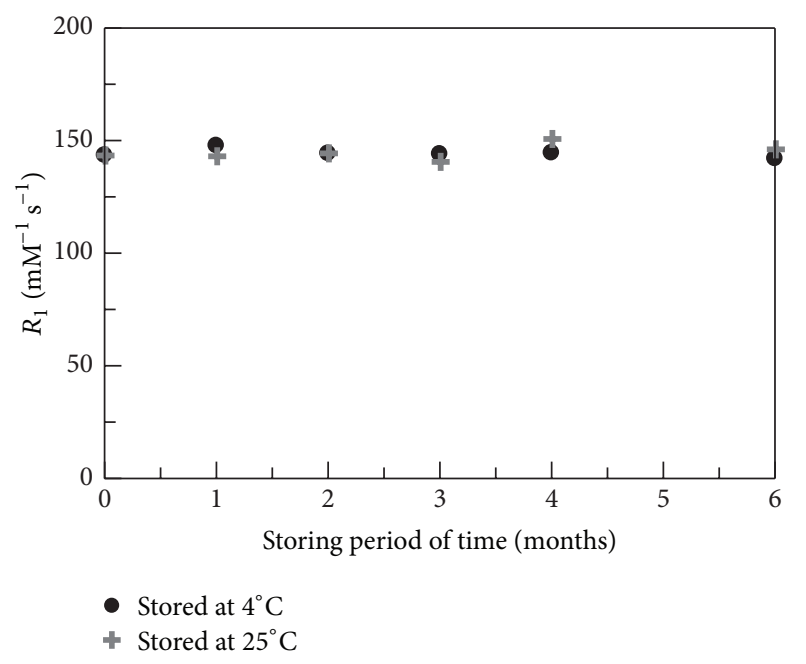

FIGURE 6: Storing period of time dependent relaxivity $R_{1}$ of MR for the solutions of dextran-coated magnetic nanoparticles stored at $4^{\circ} \mathrm{C}$ and $25^{\circ} \mathrm{C}$, respectively.

solution is found to be $3.17 \%$ via (1). The observed reduction in the $\chi_{\mathrm{ac}}$ of the reagent in Figure 5 demonstrates that the anti-CEA functionalized $\mathrm{Fe}_{3} \mathrm{O}_{4}$ magnetic nanoparticles are capable of binding with CEA molecules.

To examine the MR stability of the $\mathrm{Fe}_{3} \mathrm{O}_{4}$ magnetic nanoparticles suspended in PBS solution, the time-evolution relaxivity of MR, for example, $R_{1}$, is detected. It is worth noting that the solution of dextran-coated $\mathrm{Fe}_{3} \mathrm{O}_{4}$ magnetic nanoparticles is separated into two groups stored at $4^{\circ} \mathrm{C}$ and $25^{\circ} \mathrm{C}$, respectively. The measured relaxivity $R_{1}$ of MR as a function of the storing period of time is shown in Figure 6. It was found that $R_{1}$ is around $145 \mathrm{mM}^{-1} \mathrm{~s}^{-1}$ for the solution of dextran-coated magnetic nanoparticles. Furthermore, the $R_{1}$ keeps unchanged for six months no matter the solution of dextran-coated magnetic nanoparticles is stored at $4^{\circ} \mathrm{C}$ or $25^{\circ} \mathrm{C}$. This means that the magnetic stability of dextrancoated $\mathrm{Fe}_{3} \mathrm{O}_{4}$ magnetic nanoparticles can be six months at least.

The anti-CEA functionalized $\mathrm{Fe}_{3} \mathrm{O}_{4}$ magnetic nanoparticles with high-stability magnetism and suspension are injected into mice implanted with colorectal tumor cells, CT26. The time-evolution MR images of the tumor are taken after the injection into mouse 1, as shown in Figure 7. The tumor is circled and indicated with an arrow. The brightness of the images at a certain instant is normalized to that of water. The normalized brightness is referred to as image contrast. The image contrast of the circled tumor is analyzed at every image-taking instant. Then, the image contrast of the circled tumor at $0 \mathrm{~h}$ is used as a reference value. The ratio in the images contrast of the circled tumor at every image-taking instant to that at $0 \mathrm{~h}$ is calculated and shown with dots in Figure 8 . The cross symbols in Figure 8 correspond to the time dependent image contrast for mouse 2 . The results for both mice definitely show that the image contrast of the colorectal tumor becomes the most significant at around 20 hours after the injection. According to the data shown in
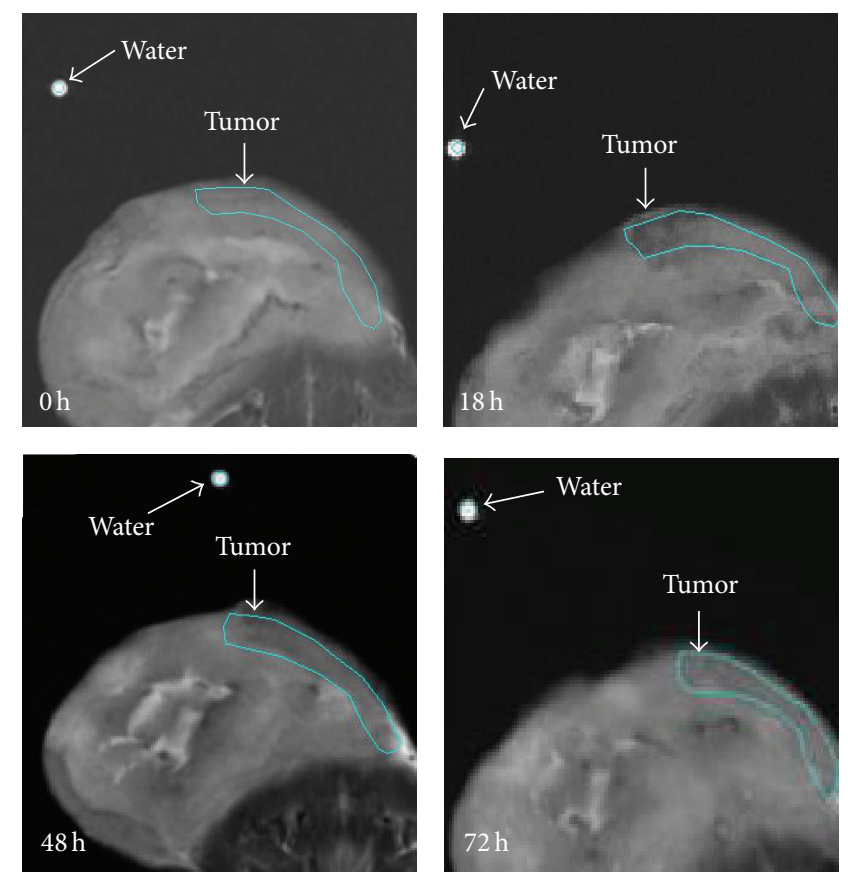

Figure 7: Temporal 3-T MR images after the injection of antibody functionalized $\mathrm{Fe}_{3} \mathrm{O}_{4}$ magnetic nanoparticles into a mouse implanted with tumor (CT-26) from 0 hour $(0 \mathrm{~h})$ to 96 hours $(96 \mathrm{~h})$.

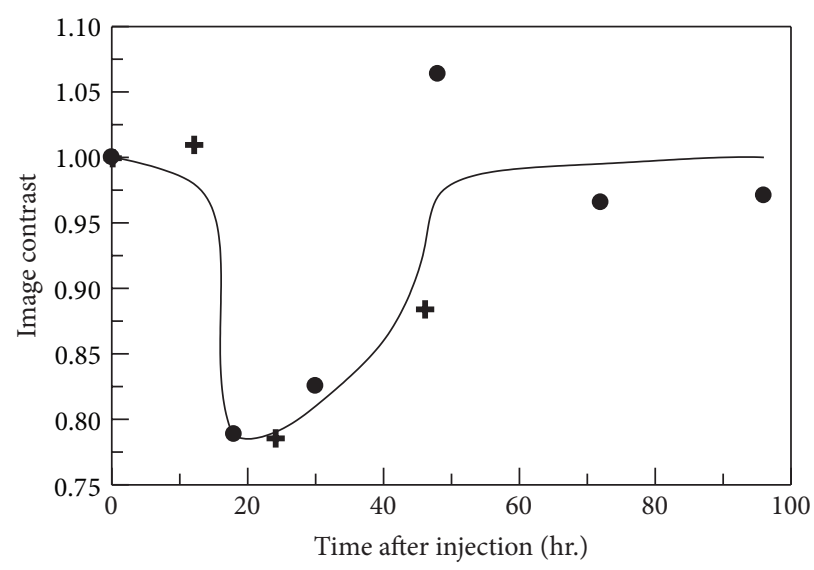

- Mouse 1

+ Mouse 2

FIGURE 8: Time dependent MR image contrast of the implanted colorectal tumor in mice after the injection of anti-CEA functionalized $\mathrm{Fe}_{3} \mathrm{O}_{4}$ magnetic nanoparticles.

Figure 8 , the brightness of the tumor is reduced by $22 \%$ at $20 \mathrm{~h}$ as compared to that at $0 \mathrm{~h}$. The brightness of the colorectal tumor recovers after 50 hours after the injection.

The reduction in the brightness of MR image for the colorectal tumor is deduced to be due to the association of anti-CEA functionalized $\mathrm{Fe}_{3} \mathrm{O}_{4}$ magnetic nanoparticles with the tumor. To prove this deduction, the biopsy of the colorectal tumors in other mice injected with anti-CEA 

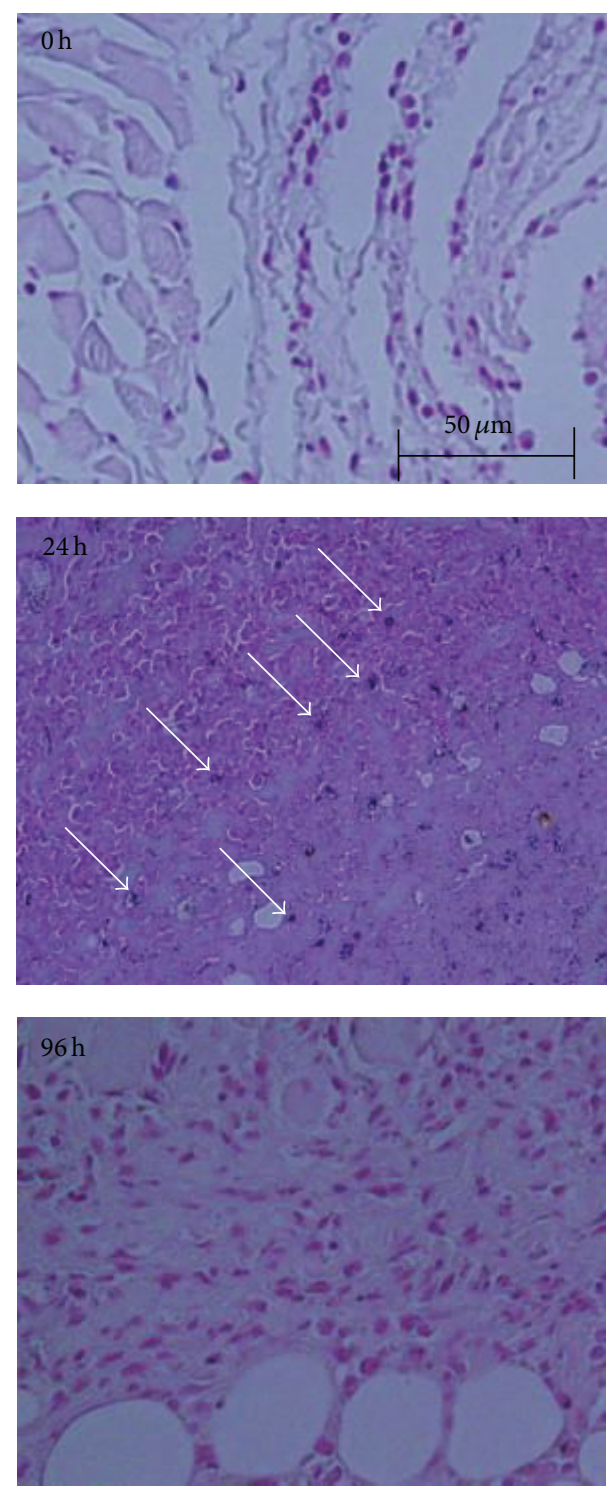

Figure 9: Temporal images of Prussian blue staining for the tumor tissue after the injection of antibody functionalized $\mathrm{Fe}_{3} \mathrm{O}_{4}$ magnetic nanoparticles into a mouse implanted with tumor (CT-26) at 0 hour (0 h), 24 hours (24 h) and 96 hours (96 h).

functionalized $\mathrm{Fe}_{3} \mathrm{O}_{4}$ magnetic nanoparticles is inspected with tissue staining. To investigate the existence of $\mathrm{Fe}$ in the tissue, Prussian blue staining is processed. The results of the Prussian blue staining for tumor tissues taken at various instants after the injection of anti-CEA functionalized $\mathrm{Fe}_{3} \mathrm{O}_{4}$ magnetic nanoparticles are shown in Figure 9. Before the injection of magnetic nanoparticles, there is no Fe-spot in the tumor tissue. The brightness of MR image of the tumor should remain unchanged; that is, image contrast $=1$. This coincides with the results shown in Figure 8. However, lots of Fe-spots can be found in the tumor tissue at 24 hours after the injection, as labeled with arrows. This implies that the brightness of the MR image of the tumor is obviously reduced; that is, image contrast $<1$, which is consistent with the result in Figure 8. At 98 hours after the injection, Fe-spot in the tissue disappears. The brightness of the MR image of the tumor should recover, as found in Figure 8 . The results in Figure 9 prove that the reduction in the brightness of MR image of the colorectal tumor is due to the association of antiCEA functionalized $\mathrm{Fe}_{3} \mathrm{O}_{4}$ magnetic nanoparticles with the tumor.

Through the careful inspection of Figure 8, significant reduction in the brightness of MR image of the colorectal tumor happens from 20 hours to 40 hours after injecting anti-CEA functionalized $\mathrm{Fe}_{3} \mathrm{O}_{4}$ magnetic nanoparticles into mice. This means that the anti-CEA functionalized magnetic nanoparticles stay on the tumor for almost 20 hours. Therefore, the suitable time widow for MR imaging of the tumor with an aid of antibody functionalized $\mathrm{Fe}_{3} \mathrm{O}_{4}$ magnetic nanoparticles is from 20 hours to 40 hours after the tail-vein injection.

\section{Conclusions}

High-stability antibody functionalized $\mathrm{Fe}_{3} \mathrm{O}_{4}$ magnetic nanoparticles are synthesized for the use of contrast medium of MR imaging. By injecting these nanoparticles into mice, target MR imaging, such as imaging colorectal tumor, is demonstrated. It is further clarified that the suitable time window for MR imaging for the target tumor is from 20 hours to 40 hours after the tail-vein injection.

\section{Conflict of Interests}

The authors declare that there is no conflict of interests regarding the publication of this paper.

\section{Acknowledgments}

This work was supported by the Ministry of Science and Technology of Taiwan, under Grant nos. 102-2112-M-003017, 102-2923-M-003-001, 102-2120-M-168-001, 102-2112-M168-001, 102-2221-E-003-008-MY2, and 101-2221-E-003-005, by the Ministry of Health and Welfare of Taiwan under Grant nos. DOH101-TD-N-111-004, DOH100-TD-N-111-008, and DOH100-TD-PB-111-TM022, and by the Ministry of Economic Affairs of Taiwan under Grant nos. 101-EC-17-A17-I1-0074, 1Z970688, and 1Z0990415 (SBIR).

\section{References}

[1] R. Kötitz, W. Weitschies, L. Trahms, W. Brewer, and W. Semmler, "Determination of the binding reaction between avidin and biotin by relaxation measurements of magnetic nanoparticles," Journal of Magnetism and Magnetic Materials, vol. 194, no. 1, pp. 62-68, 1999.

[2] K. Enpuku, D. Kuroda, A. Ohba et al., "Biological immunoassay utilizing magnetic marker and high $\mathrm{T}_{c}$ superconducting quantum interference device magnetometer," Japanese Journal of Applied Physics, vol. 42, no. 12, pp. L1436-L1438, 2003.

[3] H. E. Horng, S. Y. Yang, Y. W. Huang, W. Q. Jiang, C.Hong, and H. C. Yang, "Nanomagnetic particles for SQUIDbased magnetically labeled immunoassay," IEEE Transactions on Applied Superconductivity, vol. 15, no. 2, pp. 668-671, 2005. 
[4] C. Hong, C. C. Wu, Y. C. Chiu, S. Y. Yang, H. E. Horng, and H. C. Yang, "Magnetic susceptibility reduction method for magnetically labeled immunoassay," Applied Physics Letters, vol. 88, no. 21, Article ID 212512, 2006.

[5] H. Krause, N. Wolters, Y. Zhang et al., "Magnetic particle detection by frequency mixing for immunoassay applications," Journal of Magnetism and Magnetic Materials, vol. 311, no. 1, pp. 436444, 2007.

[6] S. H. Liao, H. C. Yang, H. E. Horng et al., "Time-dependent phase lag of biofunctionalized magnetic nanoparticles conjugated with biotargets studied with alternating current magnetic susceptometor for liquid phase immunoassays," Applied Physics Letters, vol. 103, Article ID 24 3703, pp. 1-3, 2013.

[7] K. W. Huang, S. Y. Yang, Y. W. Hong et al., "Feasibility studies for assaying $\alpha$-fetoprotein using antibody-activated magnetic nanoparticles," International Journal of Nanomedicine, vol. 7, pp. 1991-1996, 2012.

[8] M. J. Chiu, S. Y. Yang, T. F. Chen et al., "New assay for old markers-plasma beta amyloid of mild cognitive impairment and Alzheimer's disease," Current Alzheimer Research, vol. 9, no. 10, pp. 1142-1148, 2012.

[9] J. K. Hsiao, M. F. Tai, H. H. Chu et al., "Magnetic nanoparticle labeling of mesenchymal stem cells without transfection agent: Cellular behavior and capability of detection with clinical $1.5 \mathrm{~T}$ magnetic resonance at the single cell level," Magnetic Resonance in Medicine, vol. 58, no. 4, pp. 717-724, 2007.

[10] K. G. Neoh and E. T. Kang, "Surface modification of magnetic nanoparticles for stem cell labeling," Soft Matter, vol. 8, no. 7, pp. 2057-2069, 2012.

[11] T. Hiroki, S. Taira, H. Katayanagi et al., "Functional magnetic nanoparticles for use in a drug delivery system," Journal of Physics, vol. 200, no. 12, Article ID 122003, 2010.

[12] H. W. Yang, M. Y. Hua, H. L. Liu, C. Y. Huang, and K. C. Wei, "Potential of magnetic nanoparticles for targeted drug delivery," Nanotechnology, Science and Applications, vol. 5, no. 1, pp. 73-86, 2012.

[13] A. Ito, M. Shinkai, H. Honda, and T. Kobayashi, "Heat-inducible TNF- $\alpha$ gene therapy combined with hyperthermia using magnetic nanoparticles as a novel tumor-targeted therapy," Cancer Gene Therapy, vol. 8, no. 9, pp. 649-654, 2001.

[14] T. Kobayashi, "Cancer hyperthermia using magnetic nanoparticles," Biotechnology Journal, vol. 6, no. 11, pp. 1342-1347, 2011.

[15] B. A. Moffat, G. Ramachandra Ready, P. McConville et al., "A novel polyacrylamide magnetic nanoparticle contrast agent for molecular imaging using MRI," Molecular Imaging, vol. 2, no. 4, pp. 324-332, 2003.

[16] B. Chertok, B. A. Moffat, A. E. David et al., "Iron oxide nanoparticles as a drug delivery vehicle for MRI monitored magnetic targeting of brain tumors," Biomaterials, vol. 29, no. 4, pp. 487496, 2008.

[17] L. Bu, J. Xie, K. Chen et al., "Assessment and comparison of magnetic nanoparticles as MRI contrast agents in a rodent model of human hepatocellular carcinoma," Contrast Media and Molecular Imaging, vol. 7, no. 4, pp. 363-372, 2012.

[18] J. Huang, X. Zhong, L. Wang, L. Yang, and H. Mao, "Improving the magnetic resonance imaging contrast and detection methods with engineered magnetic nanoparticles," Theranostics, vol. 2, no. 1, pp. 86-102, 2012.

[19] Y. X. Wang, "Superparamagnetic iron oxide based MRI contrast agents: current status of clinical application," Quantitative Imaging in Medicine and Surgery, vol. 1, no. 1, pp. 35-40, 2011.
[20] A. Bogdanov Jr. and M. L. Mazzanti, "Molecular magnetic resonance contrast agents for the detection of cancer: past and present," Seminars in Oncology, vol. 38, no. 1, pp. 42-54, 2011.

[21] L. A. Carbonaro, F. Pediconi, N. Verardi, R. M. Trimboli, M. Calabrese, and F. Sardanelli, "Breast MRI using a high-relaxivity contrast agent: an overview," American Journal of Roentgenology, vol. 196, no. 4, pp. 942-955, 2011.

[22] W. Cheng, Y. Ping, Y. Zhang, K. Chuang, and Y. Liu, "Magnetic resonance imaging (MRI) contrast agents for tumor diagnosis," Journal of Healthcare Engineering, vol. 4, no. 1, pp. 23-46, 2013.

[23] W. Jiang, H. C. Yang, S. Y. Yang et al., "Preparation and properties of superparamagnetic nanoparticles with narrow size distribution and biocompatible," Journal of Magnetism and Magnetic Materials, vol. 283, no. 2-3, pp. 210-214, 2004.

[24] C. Y. Hong, C. C. Wu, Y. C. Chiu, S. Y. Yang, H. E. Horng, and H. C. Yang, "Magnetic susceptibility reduction method for magnetically labeled immunoassay," Applied Physics Letters, vol. 88, no. 21, Article ID 212512, 2006.

[25] J. J. Chieh, S.-Y. Yang, H.-E. Horng et al., "Immunomagnetic reduction assay using high- $\mathrm{T}_{c}$ superconducting-quantuminterference-device-based magnetosusceptometry," Journal of Applied Physics, vol. 107, no. 7, Article ID 074903, 2010.

[26] C. C. Yang, S. Y. Yang, H. H. Chen et al., "Effect of moleculeparticle binding on the reduction in the mixed-frequency alternating current magnetic susceptibility of magnetic bioreagents," Journal of Applied Physics, vol. 112, no. 2, p. 024704, 2012. 

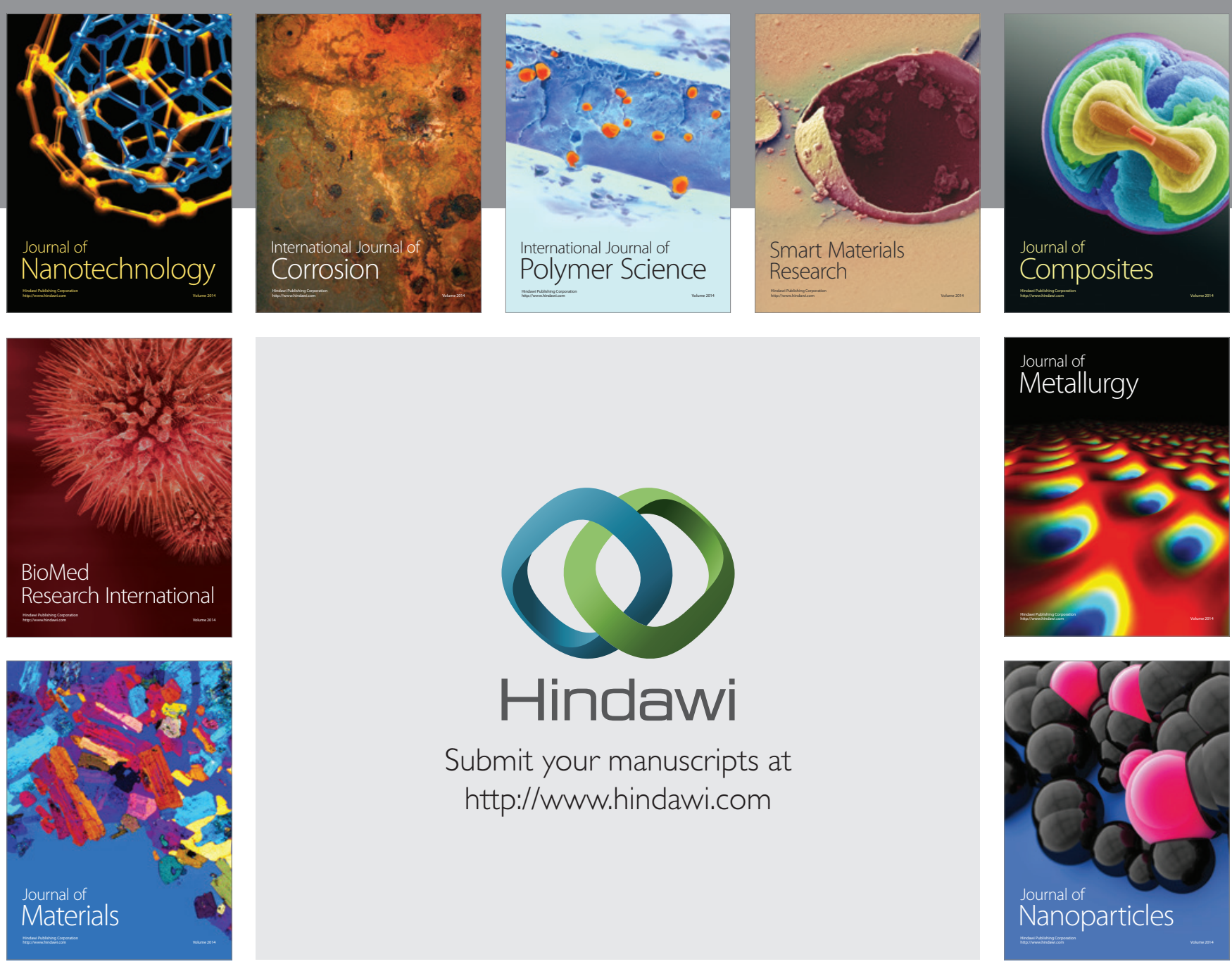

Submit your manuscripts at http://www.hindawi.com
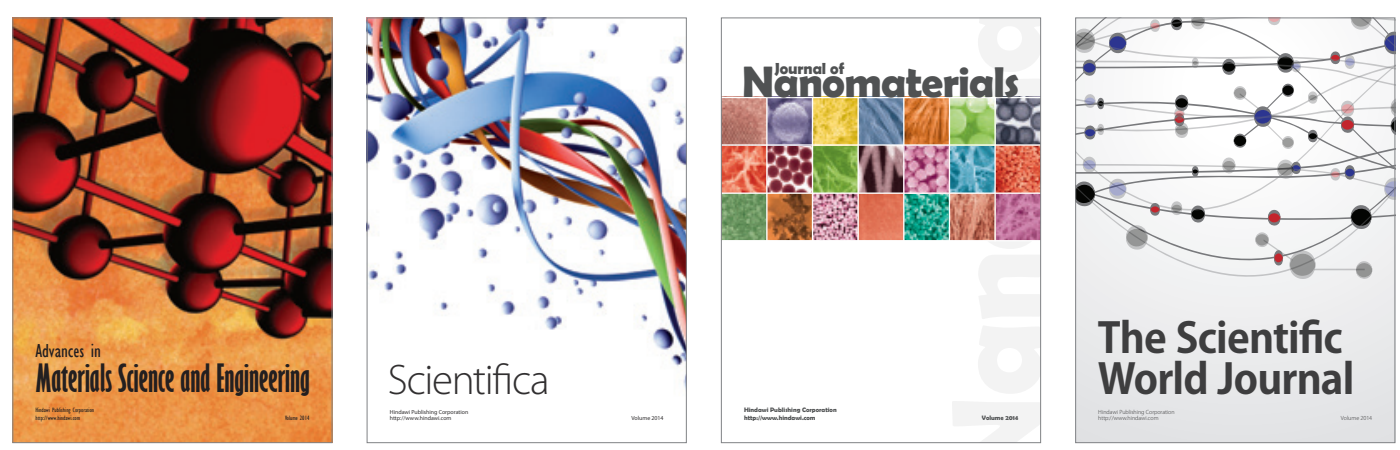

\section{The Scientific World Journal}
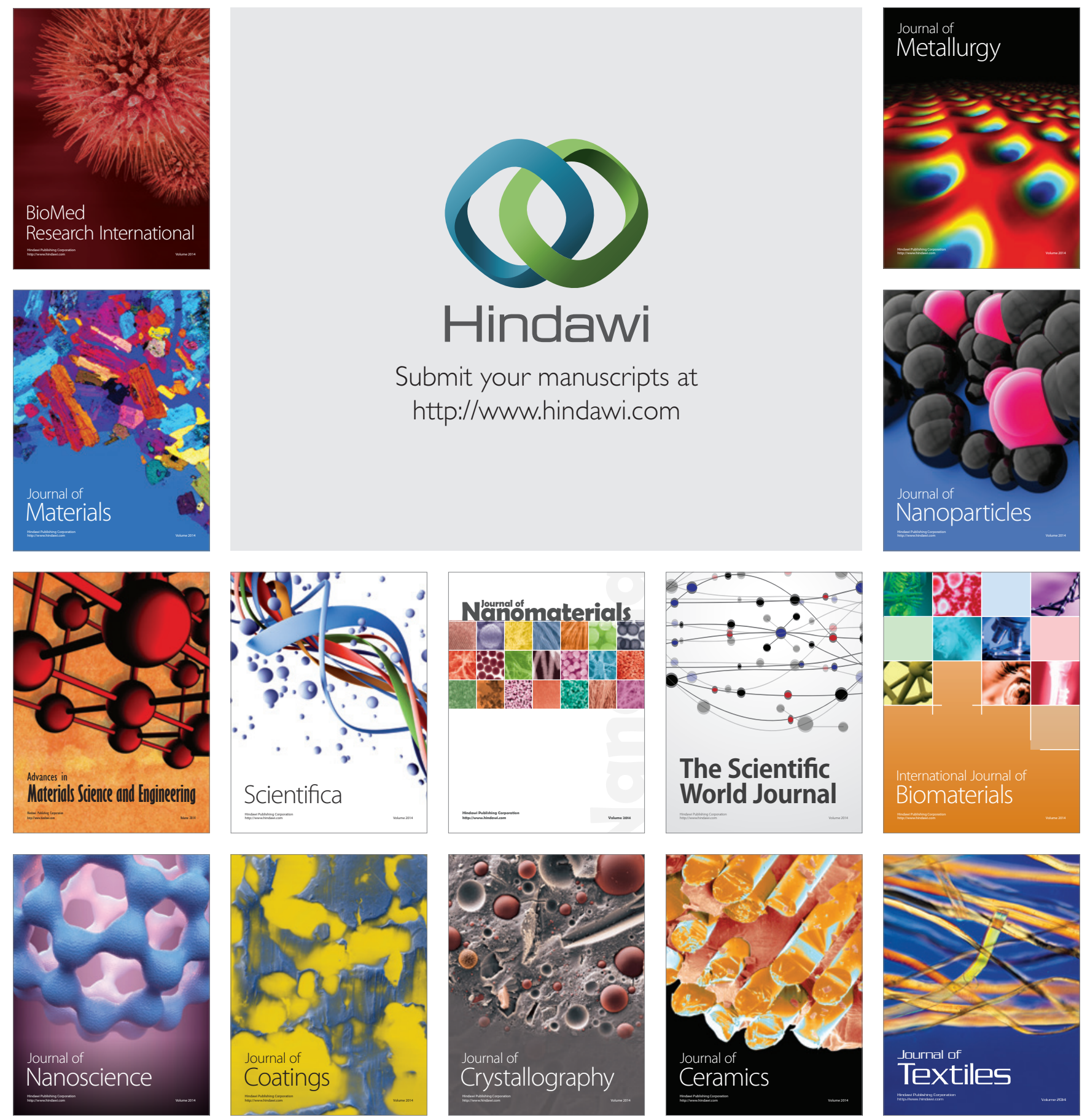